\title{
On the Nonlinear Effects of Magnetoacoustic Perturbations in a Perfectly Conducting Viscous and Thermoconducting Gas
}

\author{
A. Perelomova* \\ Gdansk University of Technology, Faculty of Applied Physics and Mathematics, \\ G. Narutowicza 11/12, 80-233 Gdansk, Poland \\ (Received June 5, 2016; in final form July 14, 2016)
}

\begin{abstract}
Nonlinear effects of planar and quasi-planar magnetosound perturbations are discussed. The plasma is assumed to be a Newtonian thermoconducting gas with infinite electrical conductivity permeated by a magnetic field orthogonal to the trajectories of gas particles. Generation of the non-wave modes (magnetoacoustic heating and streaming) in the field of periodic and aperiodic magnetoacoustic perturbations is discussed. The results are compared with acoustic heating and streaming in an nonmagnetized Newtonian gas. In contrast with a Newtonian gas, the magnetoacoustic forces of heating and streaming are not proportional to the summary attenuation. The magnetoacoutic force of streaming is always smaller than the Newtonian one, but that of heating may take larger or smaller value in dependence on the magnetic strength.
\end{abstract}

DOI: 10.12693/APhysPolA.130.727

PACS/topics: $43.25 . \mathrm{Nm}$

\section{Introduction}

There has been much discussion in the literature during the past years which concern magnetohydrodynamic phenomena in conducting fluids. They are of importance in many applications of cosmic physics, geophysics, plasma physics, physics of controlled thermonuclear fusion and hypersonic aerodynamics. The understanding that sound velocity in electrically conducting fluid varies in presence of magnetic field comes from the 50th years of the last century $[1,2]$. It has been established that the finite conductivity introduces dispersion and absorption of sound planar waves which propagates perpendicular to the magnetic field [3]. Many studies are devoted to magneto-hydrodynamic waves in the perfectly conducting gases, and also to their nonlinear effects. For nonlinear systems, we do not have the luxury of exact solutions. Nevertheless, there are evident successes on the way of establishing of complete exact solutions to the system of PDEs describing one-dimensional unsteady planar and cylindrically symmetric motions in magnetohydrodynamics (MHD), involving solutions with discontinuities [4-6]. Geffen has developed a consistent nonlinear first-order theory for the magnetogasdynamic two-dimensional and axisymmetric flow of an inviscid conductor around slender bodies [7]. Structure of MHD shock waves in a viscous compressible plasma was studied in Ref. [8], and that in a viscous non-ideal gas in Ref. [9]. Inclusion of radiation imposes solution of a complex set of integrodifferential equations which take into account the frequency dependence of the radiation field. Propagation of weak discontinuities and their formation into shock waves in a thermally-radiative ideal plasma were investigated in

\footnotetext{
*e-mail: anpe@mif.pg.gda.pl
}

Ref. [10]. The effects of thermal radiation on shock wave were studied in detail, among other, in Refs. [6,11], and the short review of problems relating to wave propagation with consideration of nonlinear phenomena may be found in this last reference. The nonlinear interaction of magnetohydrodynamic waves has been considered by numerous authors [12-14]. Ponomarev has attracted attention to nonlinear transfer of energy between magnetohydrodynamic waves and other types of waves in plasma. Threewave interactions of kinetic Alfvén and magnetosound waves were studied in Ref. [15]. Amplification of Alfvén wave due to nonlinear interaction with a magnetoacoustic wave was discovered recently in Ref. [16]. The review by Ballai summarizes knowledge on nonlinear waves in solar plasmas [17]. It concerns also nonlinear resonant waves.

Detection and identification of acoustic modes in the solar corona have revived interest in their application to coronal seismology. Dissipation of these modes is difficult to explain by linear damping ([18] and references therein). The nonlinear theory is required, which is able to predict nonlinear losses in magnetoacoustic energy and momentum and their transfer to non-wave motions. This study considers magnetoacoustic heating and streaming in their conventional meaning, as entropy and vortex motions which follow the propagation of sound in a nonlinear flow with attenuation $[19,20]$. As far as the author knows, magnetoacoustic heating and streaming are still unresolved issues in magnetic hydrodynamics. The method which has been applied by the author in a number of hydrodynamical problems concerning damping, dispersive, non-equilibrium flows or those over the non-uniform media, gives a simple and clear sequence of actions to implement all objectives listed hereafter (see, for example, Refs. [21-23]). The description of nonlinear interaction of different types MHD motions imposes resolution of some issues: 
(1) to determine the linear types of motion, or MHD modes, as links between specific perturbations;

(2) to derive the leading-order equations which describe interaction of modes in weakly nonlinear flow;

(3) to solve them with a required precision making use of physical context of a problem.

We consider a weak nonlinearity. This means that the effects of nonlinearity may be obtained as corrections to the linear results. The main idea is to determine modes in a weakly nonlinear flow making use of links of specific perturbations in a linear flow and to establish linear projectors which eliminate all foreign modes in the linear parts of dynamic equations. This gives possibility to derive a set of weakly nonlinear coupling evolution equations, and to correct links of specific perturbations in a weakly nonlinear flow. The procedure appoints a recurrent sequence of actions to obtain results as series in powers of the Mach number $M$ with any desired accuracy.

In this study, we consider an unbounded volume of a gas in a magnetic field. All evaluations are provided with accuracy up to quadratic nonlinear terms, that is, up to terms proportional to $M^{2}$. The corrected nonlinear links correspondent to that in the Riemann wave will be established, and equations describing generation of the secondary modes in the dominative magnetocoustic field, will be derived and discussed in Sect. 3 (effects of planar sound) and in Sect. 4 (magnetoacoustic streaming in the field of quasi-planar sound wave).

\section{Decomposition of sound and non-wave modes in a planar flow}

\subsection{Basic equations describing motion} of a perfectly conducting gas

In this section, we derive the weakly nonlinear equations governing a planar flow of a gas along $x$-axis. Absorption due to shear viscosity of plasma $\eta$ and its thermal conduction $\sigma$, is taken into account. We assume the electrical conductivity to be infinite and the magnetic field $\boldsymbol{H}=(0,0, H(x, t))$ with the magnetic field strength $H$, orthogonal to the trajectories of gas particles, that is, to their velocity $\boldsymbol{v}=(v(x, t), 0,0)$. The starting point represents conservation equations [24]:

$$
\frac{\partial \rho}{\partial t}+\frac{\partial(\rho v)}{\partial x}=0
$$

for mass,

$$
\rho\left(\frac{\partial v}{\partial t}+v \frac{\partial v}{\partial x}\right)+\frac{\partial p}{\partial x}+\frac{\partial h}{\partial x}=\frac{4 \eta}{3} \frac{\partial^{2} v}{\partial x^{2}},
$$

for momentum,

$$
\rho T\left(\frac{\partial s}{\partial t}+v \frac{\partial s}{\partial x}\right)=\frac{\partial}{\partial x}\left(\sigma \frac{\partial T}{\partial x}\right)+\frac{4 \eta}{3}\left(\frac{\partial v}{\partial x}\right)^{2},
$$

for entropy $s$, and

$$
\frac{\partial h}{\partial t}+v \frac{\partial h}{\partial x}+2 h \frac{\partial v}{\partial x}=0
$$

for the magnetic pressure $h$,

$$
h=\mu H^{2} / 2 \text {. }
$$

$t$ denotes time, $\rho, p, T$ are total density, total pressure, and temperature of a gas, respectively, $\mu$ is the magnetic permeability. Supposing that the viscosity and thermal conductivity are fixed fractions of their non-magnetized values and obey power-law dependence on temperature, we may consider them in a form:

$$
\eta=\kappa T^{\alpha}, \quad \sigma=\chi T^{\alpha} .
$$

In a fully ionized plasma, $\alpha=5 / 2$ and $\kappa, \chi$ are some constants [25-27]. Equation (4) follows from the equations:

$$
\frac{\partial \boldsymbol{H}}{\partial t}-\nabla \times(\boldsymbol{v} \times \boldsymbol{H})=\mathbf{0}, \quad \boldsymbol{\nabla} \cdot \boldsymbol{H}=0
$$

and condition of perpendicularity of magnetic field and velocity of a gas, $\boldsymbol{H} \cdot \boldsymbol{v}=0[5]$.

\subsection{Modes in a flow of infinitely-small magnitude. Extracting of the specific modes from the total perturbations}

All variables represent a sum of unperturbed quantity, marked by subscript $0\left(v_{0}=0\right)$, and a disturbance, which is primed. We make use of caloric and thermal equations of state of an ideal gas

$$
e=C_{V} T=\frac{p}{(\gamma-1) \rho},
$$

where $e$ denotes the internal energy, $C_{V}$ is the heat capacity per unit mass under constant volume, $\gamma=C_{p} / C_{V}$ is the ratio of specific heats under constant pressure and constant density, and of thermodynamic identity $T \mathrm{~d} s=\mathrm{d} e+p \mathrm{~d}\left(\rho^{-1}\right)$. The leading-order system of $\mathrm{dy}-$ namic equations follows from Eqs. (1)-(4), it includes quadratic nonlinear terms:

$$
\begin{aligned}
& \frac{\partial \rho^{\prime}}{\partial t}+\rho_{0} \frac{\partial v}{\partial x}=-\rho_{0}^{\prime} \frac{\partial v}{\partial x}-v \frac{\partial \rho^{\prime}}{\partial x} \\
& \frac{\partial v}{\partial t}-\delta_{1} \frac{\partial^{2} v}{\partial x^{2}}+\frac{1}{\rho_{0}} \frac{\partial p^{\prime}}{\partial x}+\frac{1}{\rho_{0}} \frac{\partial h^{\prime}}{\partial x}=-v \frac{\partial v}{\partial x}+\frac{\rho^{\prime}}{\rho_{0}^{2}} \frac{\partial p^{\prime}}{\partial x} \\
& +\frac{\rho^{\prime}}{\rho_{0}^{2}} \frac{\partial h^{\prime}}{\partial x}-\frac{\delta_{1} \rho^{\prime}}{\rho_{0}} \frac{\partial^{2} v}{\partial x^{2}}+\frac{\alpha \delta_{1}\left(\gamma p^{\prime}-c_{0}^{2} \rho^{\prime}\right)}{\gamma p_{0}} \frac{\partial^{2} v}{\partial x^{2}}, \\
& \frac{\partial p^{\prime}}{\partial t}-\frac{\gamma \delta_{2}}{\gamma-1} \frac{\partial^{2} p^{\prime}}{\partial x^{2}}+\frac{\delta_{2} c_{0}^{2}}{\gamma-1} \frac{\partial^{2} \rho^{\prime}}{\partial x^{2}}+c_{0}^{2} \rho_{0} \frac{\partial v}{\partial x}= \\
& -v \frac{\partial p^{\prime}}{\partial x}-\gamma p^{\prime} \frac{\partial v}{\partial x}+\delta_{1}(\gamma-1) \rho_{0}\left(\frac{\partial v}{\partial x}\right)^{2} \\
& \quad-\frac{\delta_{2}}{\rho_{0}(\gamma-1)} \frac{\partial^{2}\left(\gamma p^{\prime} \rho^{\prime}-c_{0}^{2} \rho^{\prime 2}\right)}{\partial x^{2}}+\frac{\alpha \delta_{2}}{(\gamma-1) \gamma p_{0}} \\
& \quad \times\left(\frac{\partial\left(\gamma p^{\prime}-c_{0}^{2} \rho^{\prime}\right)}{\partial x}\right)^{2}, \\
& \frac{\partial h^{\prime}}{\partial t}+2 h_{0} \frac{\partial v}{\partial x}=-v \frac{\partial h^{\prime}}{\partial x}-2 h^{\prime} \frac{\partial v}{\partial x},
\end{aligned}
$$

where $\delta_{1}, \delta_{2}$ are coefficients responsible for viscous and thermal effects, and $c_{0}$ is the speed of sound of infinitely small magnitude $c\left(c_{0}\right.$ relates to an ideal gas and is evaluated at unperturbed thermodynamic state $p_{0}, \rho_{0}$ in the absence of magnetic field):

$$
\delta_{1}=\frac{4 \kappa T_{0}^{\alpha}}{3 \rho_{0}}, \quad \delta_{2}=\left(\frac{1}{C_{v}}-\frac{1}{C_{p}}\right) \frac{\chi T_{0}^{\alpha}}{\rho_{0}}, \quad c=\sqrt{\frac{\gamma p}{\rho}} .
$$


Establishment of the dispersion relations is the primary procedure in all linear and weakly nonlinear fluid flows. The dispersion relations describing all independent modes follow from the linearized version of Eqs. (5)-(8). Studies of planar motions of infinitely-small magnitude begin usually by representing all perturbations as a sum of planar waves proportional to $\exp (\mathrm{i} \omega(k) t-\mathrm{i} k x)$, where $k$ designates the wave number of any individual planar wave

$$
f^{\prime}(x, t)=\int_{-\infty}^{\infty} \widetilde{f}(k) \exp (\mathrm{i} \omega(k) t-\mathrm{i} k x) \mathrm{d} k
$$

$(\widetilde{f}(k) \exp (i \omega(k) t)=\widetilde{f}(k, t)$ denotes the Fourier transform of $\left.f^{\prime}(x, t), \tilde{f}(k, t)=\frac{1}{2 \pi} \int_{-\infty}^{\infty} f(x, t) \mathrm{e}^{\mathrm{i} k x} \mathrm{~d} x\right)$. In a planar flow of a magnetic fluid, the dispersion relations take the leading-order form

$$
\begin{aligned}
& \omega_{1,2}= \pm c_{m, 0} k+\frac{\mathrm{i}}{2}\left(\delta_{1}+\frac{c_{0}^{2}}{c_{m, 0}^{2}} \delta_{2}\right) k^{2}, \\
& \omega_{3}=\mathrm{i}\left(\frac{\delta_{2}}{\gamma-1}+\frac{\delta_{2}\left(c_{m, 0}^{2}-c_{0}^{2}\right)}{c_{m, 0}^{2}}\right) k^{2}, \quad \omega_{4}=0,
\end{aligned}
$$

where

$$
c_{m}=\sqrt{c^{2}+c_{\mathrm{A}}^{2}}, \quad c_{\mathrm{A}}=\sqrt{2 h / \rho}
$$

designate the magnetosonic speed and the Alfvén speed, respectively, and $c_{m, 0}$ denotes $c_{m}$ at the unperturbed state $p_{0}, \rho_{0}$.

The first two roots $\omega_{1}, \omega_{2}$ correspond to the magnetosonic waves of different directions of propagation (fast MHD waves), the third root $\omega_{3}$ corresponds to the entropy mode, and the last one, $\omega_{4}$, corresponds to the Alfvén wave in the flow where magnetic field is perpendicular to the particles velocity. The first three roots in Eqs. (9) are calculated with accuracy up to terms proportional to the first powers of thermoviscous coefficients $\delta_{1}, \delta_{2}$. They determine links of the Fourier-transforms of specific perturbations inside every mode, or, in the other words, eigenvectors of the correspondent linear matrix operator. It is remarkable that two last dispersion relations are zero in a fluid without thermal conduction. In this case, there are two degenerate eigenvalues and more than one linearly independent eigenvectors corresponding to each of them. Thermal conductivity eliminates this degeneracy. The total perturbation is represented by a sum of specific disturbances (we can say, eigenvectors inherent to these eigenvalues) as follows:

$$
\begin{aligned}
v & =\sum_{i=1}^{4} v_{i}=\frac{c_{m, 0}}{\rho_{0}} \rho_{1}^{\prime}-\left(\frac{\delta_{1}}{2 \rho_{0}}+\frac{\delta_{2} c_{0}^{2}}{2 \rho_{0} c_{m, 0}^{2}}\right) \frac{\partial \rho_{1}^{\prime}}{\partial x} \\
& -\frac{c_{m, 0}}{\rho_{0}} \rho_{2}^{\prime}-\left(\frac{\delta_{1}}{2 \rho_{0}}+\frac{\delta_{2} c_{0}^{2}}{2 \rho_{0} c_{m, 0}^{2}}\right) \frac{\partial \rho_{2}^{\prime}}{\partial x} \\
- & \frac{\delta_{2}\left[\gamma c_{m, 0}^{2}-c_{0}^{2}(\gamma-1)\right]}{c_{m, 0}^{2}(\gamma-1) \rho_{0}} \frac{\partial \rho_{3}^{\prime}}{\partial x},
\end{aligned}
$$

$$
\begin{aligned}
p^{\prime} & =\sum_{i=1}^{4} p_{i}^{\prime}=c_{0}^{2} \rho_{1}^{\prime}-\delta_{2} \frac{c_{0}^{2}}{c_{m, 0}} \frac{\partial \rho_{1}^{\prime}}{\partial x}+c_{0}^{2} \rho_{2}^{\prime}+\delta_{2} \frac{c_{0}^{2}}{c_{m, 0}} \frac{\partial \rho_{2}^{\prime}}{\partial x} \\
& -\left(c_{m, 0}^{2}-c_{0}^{2}\right) \rho_{3}^{\prime}+\frac{c_{0}^{2}}{\gamma} \rho_{4}^{\prime}, \\
h^{\prime} & =\sum_{i=1}^{4} h_{i}^{\prime}=\left(c_{m, 0}^{2}-c_{0}^{2}\right) \rho_{1}^{\prime}+\left(c_{m, 0}^{2}-c_{0}^{2}\right) \rho_{2}^{\prime} \\
& +\left(c_{m, 0}^{2}-c_{0}^{2}\right) \rho_{3}^{\prime}-\frac{c_{0}^{2}}{\gamma} \rho_{4}^{\prime} .
\end{aligned}
$$

Index in summation $i$ designates the ordering number of specific mode. In a thermoconducting MHD flow, the entropy mode is isobaric in the sense that summary pressure, which consists of thermodynamic and magnetic parts, keeps constant. This applies also to the Alfvén mode. We may readily establish operator rows which distinguish the specific excess density correspondent to any mode from the total vector of perturbations. In particular, rows which distinguish excess densities corresponding to the third and fourth roots,

$$
P_{3}\left(\begin{array}{c}
\rho^{\prime} \\
v \\
p^{\prime} \\
h^{\prime}
\end{array}\right)=\rho_{3}^{\prime}, \quad P_{4}\left(\begin{array}{c}
\rho^{\prime} \\
v \\
p^{\prime} \\
h^{\prime}
\end{array}\right)=\rho_{4}^{\prime},
$$

take the forms

$$
\begin{gathered}
P_{3}=\left(\begin{array}{c}
\frac{c_{0}^{2}}{\gamma c_{m, 0}^{2}-(\gamma-1) c_{0}^{2}} \\
-\frac{\delta_{2} \rho_{0} c_{0}^{2}}{c_{m, 0}^{4}} \frac{\partial}{\partial x} \\
-\frac{1}{c_{m, 0}^{2}} \\
\frac{(\gamma-1) c_{0}^{2}}{c_{m, 0}^{2}\left(\gamma c_{m, 0}^{2}-(\gamma-1) c_{0}^{2}\right)}
\end{array}\right)^{\mathrm{T}}, \\
P_{4}=\left(\begin{array}{c}
\frac{\gamma\left(c_{m, 0}^{2}-c_{0}^{2}\right)}{\gamma c_{m, 0}^{2}-(\gamma-1) c_{0}^{2}} \\
0 \\
0 \\
\gamma \\
-\frac{\gamma}{\gamma c_{m, 0}^{2}-(\gamma-1) c_{0}^{2}}
\end{array}\right)^{\mathrm{T}} .
\end{gathered}
$$

They are also evaluated with accuracy up to terms proportional to $\delta_{1}, \delta_{2}$. When $P_{3}, P_{4}$ apply at the linearized system (5)-(8), they reduce all therms of the foreign modes and yield the linear dynamic equations for $\rho_{3}^{\prime}$ and $\rho_{4}^{\prime}$.

In the following section, we consider the nonlinear interaction of sound with two non-wave modes in the planar flow. The nonlinear generation of the vortex mode in the field of magnetosound perturbations, which exists only in two- or three-dimensional flow, will be considered in Sect. 4.

\section{Nonlinear effects of planar magnetoacoustic waves}

Application of $P_{3}$ and $P_{4}$ at the system (5)-(8), which includes quadratic nonlinear terms, distributes them between dynamic equations properly. The nonlinear therms contain cross contributions of all modes, and they may be selected in accordance to the physical meaning 
of a problem. Usually, nonlinear effects of sound are of interest, and only acoustic terms are considered among all variety of nonlinear ones. These acoustic nonlinear terms form "acoustic forces" of the secondary modes.

\subsection{Nonlinear corrections in links of acoustic perturbations}

For proper description of the nonlinear effects of sound, the linear links which are determined by eigenvectors of the correspondent matrix operator, should be completed by the leading-order nonlinear terms which do not include thermoviscous coefficients. Without loss of generality, progressive in the positive direction of axis $x$ magnetoacoustic mode is considered. It corresponds to $\omega_{1}$ from Eqs. (9). The relative eigenvector in inviscid and non-heat conducting gas takes the form

$$
\psi_{1}=\left(\begin{array}{c}
\rho_{1}^{\prime} \\
v_{1} \\
p_{1}^{\prime} \\
h_{1}^{\prime}
\end{array}\right)=\left(\begin{array}{c}
\frac{\rho_{0}}{c_{m, 0}} \\
1 \\
\frac{\rho_{0} c_{0}^{2}}{c_{m, 0}} \\
\frac{\rho_{0}\left(c_{m, 0}^{2}-c_{0}^{2}\right)}{c_{m, 0}}
\end{array}\right) v_{1} .
$$

The vector with unknown constants $A, B, C$,

$$
\psi_{1, n}=\left(\begin{array}{llll}
A & 0 & B & C
\end{array}\right)^{\mathrm{T}} v_{1}^{2},
$$

should be added to $\psi_{1}$ in order to yield four equivalent leading-order dynamic nonlinear equations for magnetoacoustic perturbations, when substituted into Eqs. (5)-(8) with zero $\delta_{1}, \delta_{2}$. Solving algebraic equations, one arrives at

$$
\begin{aligned}
& A=\frac{c_{m, 0}^{2}-c_{0}^{2}(\gamma-2)}{4 c_{m, 0}^{4}} \rho_{0}, \\
& B=\frac{c_{0}^{2}\left[c_{m, 0}^{2}(2 \gamma-1)-c_{0}^{2}(\gamma-2)\right]}{4 c_{m, 0}^{4}} \rho_{0}, \\
& C=\frac{\left(c_{m, 0}^{2}-c_{0}^{2}\right)\left[3 c_{m, 0}^{2}-(\gamma-2) c_{0}^{2}\right]}{4 c_{m, 0}^{4}} \rho_{0} .
\end{aligned}
$$

These constants coincide with the well-known nonlinear corrections which make the progressive Riemann wave isentropic [19]. If $h_{0}$ tends to zero, $A=-\frac{(\gamma-3) \rho_{0}}{4 c_{0}^{2}}$, $B=\frac{\gamma+1}{4} \rho_{0}$, and $C=0$. The equation governing velocity in the first magnetoacoustic planar wave which propagates in the positive direction of axis $x$, takes the form

$$
\frac{\partial v_{1}}{\partial t}+c_{m, 0} \frac{\partial v_{1}}{\partial x}+\varepsilon_{m} v_{1} \frac{\partial v_{1}}{\partial x}=0
$$

where

$$
\varepsilon_{m}=\frac{3 c_{m, 0}^{2}+c_{0}^{2}(\gamma-2)}{2 c_{m, 0}^{2}} .
$$

Equation (18) coincides with the Earnshaw equation when $h_{0}$ tends to zero and hence $\varepsilon_{m}$ tends to $\varepsilon=$ $\frac{\gamma+1}{2}[19]$. It describes nonlinear propagation of the progressive wave in an ideal gas, including waves with discontinuity. Waveforms with discontinuity may be established by use of conservation of total magnetosound momentum. Equation (18) has been derived and used for description of propagation of a saw-tooth impulse in Ref. [5]
(Eq. (12) therein with $m=0$, which corresponds to the planar geometry of a flow).

The equation which accounts for nonlinear and thermoviscous effects, recalls the Burgers equation [19]:

$$
\begin{aligned}
\frac{\partial v_{1}}{\partial t}+c_{m, 0} \frac{\partial v_{1}}{\partial x}+\varepsilon_{m} v_{1} \frac{\partial v_{1}}{\partial x} \\
-\frac{1}{2}\left(\delta_{1}+\frac{c_{0}^{2}}{c_{m, 0}^{2}} \delta_{2}\right) \frac{\partial^{2} v_{1}}{\partial x^{2}}=0 .
\end{aligned}
$$

Equation (19) coincides with that derived in Ref. [28] for any direction of the magnetic field (this is the case $\theta=\pi / 2$ in Eq. (15) therein with zero heating and radiative cooling of the plasma). Chin and co-authors in Ref. [28] include heating and radiative cooling but consider perturbations over constant background. The validity of this approach should be carefully investigated in view of that the external source makes the background temperature nonuniform. Namely, this follows from the energy balance for the thermoconducting fluid in the zero order. The uniformity of the background may essentially affect the wave dynamics, especially at the low frequencies. Thermoviscous attenuation of the magnetoacoustic perturbation in its part which relates to the thermal conduction, is smaller as compared with a non-magnetized Newtonian flow where the total attenuation equals $\delta_{1}+\delta_{2}$. Damping of magnetoacoustic wave depends in general on magnetic pressure, $h_{0}$, by means of $c_{m, 0}$. Solutions of Eq. (19) may be established by the well-known methods suitable for the Burgers equation. In particular, it transforms into the linear diffusion equation by the HopfCole transformation [19]. The acoustic Reynolds number is also dependent on the magnetic strength. It may be readily concluded that the acoustic Reynolds number is always larger in the magnetoacoustic wave as compared to sound for perturbations with equal Mach numbers and frequencies.

\subsection{Enhancement of the foreign modes}

in the field of magnetoacoustic waves of high intensity

The progressive magnetosonic mode, which is an analogue of the Riemann wave in an ideal inviscid gas, is represented in the leading order by a sum $\psi_{1}+\psi_{1, n}$. The projecting rows $P_{3}$ and $P_{4}$ point a way of linear combining of Eqs. (5)-(8) in order to eliminate foreign terms in the linear part of equations which describe dynamics of $\rho_{3}^{\prime}$ or $\rho_{4}^{\prime}$. Among variety of quadratic nonlinear terms, only these ones belonging to the first progressive mode, will be kept. This corresponds to the dominative magnetoacoustic perturbation which propagate in the positive direction of axis $x$. The thermoviscous nonlinear terms, which originate from the thermoviscous nonlinear magnetoacoustic terms in the initial Eqs. (5)-(8), and from the viscous terms in the projecting rows, form the "magnetoacoustic forces" of the secondary modes. As the result of application of $P_{3}$, one arrives at equation which governs an excess density in the entropy mode 


$$
\begin{aligned}
& \frac{\partial \rho_{3}^{\prime}}{\partial t}-\left(\frac{\delta_{2}}{\gamma-1}+\frac{\delta_{2}\left(c_{m, 0}^{2}-c_{0}^{2}\right)}{c_{m, 0}^{2}}\right) \frac{\partial^{2} \rho_{3}^{\prime}}{\partial x^{2}}= \\
& \quad-\rho_{0}\left(2 c_{m, 0}^{6}\left(\gamma c_{m, 0}^{2}-c_{0}^{2}(\gamma-1)\right)\right)^{-1}\left(c _ { 0 } ^ { 4 } c _ { m , 0 } ^ { 2 } \left(\delta_{2}(5-8 \gamma)\right.\right. \\
& \left.\quad+\delta_{1}(\gamma-1)\right)(\gamma-2)+2 c_{m, 0}^{6} \delta_{1}(\gamma-1) \gamma \\
& \quad+4 c_{0}^{6} \delta_{2}(\gamma-1)(\gamma-2)+c_{0}^{2} c_{m, 0}^{4}\left(-3 \delta_{1}(\gamma-1)^{2}\right. \\
& \left.\left.\quad+\delta_{2} \gamma(4 \gamma-9)\right)\right)\left(\frac{\partial v_{1}}{\partial x}\right)^{2}-\rho_{0}\left(2 c _ { m , 0 } ^ { 6 } \left(\gamma c_{m, 0}^{2}\right.\right. \\
& \left.\left.-c_{0}^{2}(\gamma-1)\right)\right)^{-1}\left(c _ { 0 } ^ { 2 } \left(-c_{m, 0}^{4}\left(\delta_{1}(\gamma-1)^{2}+\delta_{2} \gamma(7-2 \gamma)\right.\right.\right. \\
& \quad+4 c_{0}^{4} \delta_{2}(\gamma-1)(\gamma-2)+c_{0}^{2} c_{m, 0}^{2}\left(\delta_{1}(\gamma-1)(\gamma-2)\right. \\
& \left.\left.+\delta_{2}\left(-6 \gamma^{2}+17 \gamma-8\right)\right)\right) v_{1} \frac{\partial^{2} v_{1}}{\partial x^{2}} \\
& \quad-\frac{\alpha c_{0}^{2} \delta_{2}(\gamma-1) \rho_{0}}{c_{m, 0}^{4}}\left(\frac{\partial v_{1}}{\partial x}\right)^{2} \cdot
\end{aligned}
$$

Equation (20) coincides with that which describes the instantaneous acoustic heating in the Newtonian flows of non-magnetized gases [29]. For periodic sound, in the leading order,

$$
\overline{\left(\frac{\partial v_{1}}{\partial x}\right)^{2}}=-\overline{v_{1} \frac{\partial^{2} v_{1}}{\partial x^{2}}},
$$

where top line denotes the temporal average over period of the acoustic wave, and Eq. (20) simplifies as

$$
\begin{gathered}
\frac{\overline{\partial \rho_{3}^{\prime}}}{\partial t}-\left(\frac{\delta_{2}}{\gamma-1}+\frac{\delta_{2}\left(c_{m, 0}^{2}-c_{0}^{2}\right)}{c_{m, 0}^{2}}\right) \frac{\overline{\partial^{2} \rho_{3}^{\prime}}}{\partial x^{2}}=F_{m, h}= \\
-\left(\frac{\left(c_{m, 0}^{2} \delta_{1}+(1+\alpha) c_{0}^{2} \delta_{2}\right)(\gamma-1) \rho_{0}}{c_{m, 0}^{4}}\right) \overline{\left(\frac{\partial v_{1}}{\partial x}\right)^{2}} .
\end{gathered}
$$

The equation which describes acoustic heating of a Newtonian gas in the absence of magnetic field (in the limit $\left.h_{0} \rightarrow 0, \alpha \rightarrow 0\right)$, takes the form [19, 21]:

$$
\begin{gathered}
\frac{\overline{\partial \rho_{3}^{\prime}}}{\partial t}-\frac{\delta_{2}}{\gamma-1} \frac{\overline{\partial^{2} \rho_{3}^{\prime}}}{\partial x^{2}}=F_{N, h}= \\
-\frac{\left(\delta_{1}+\delta_{2}\right)(\gamma-1) \rho_{0}}{c_{0}^{2}} \overline{\left(\frac{\partial v_{1}}{\partial x}\right)^{2}} .
\end{gathered}
$$

It always describes the isobaric increase of temperature of an ideal gas, which corresponds to variation in its density, $\rho_{3}^{\prime}$. The acoustic force of acoustic heating is proportional to the total attenuation, $\delta_{1}+\delta_{2}$. Equation (22) is wellknown in the theory of non-linear acoustics [30]. As compared with Eq. (22), the equation which governs magnetoacoustic heating, contains smaller part responsible for viscosity in the acoustic force in its right-hand side. As for the coefficient by part which associates with thermal conduction, it is larger than non-magnetized value, if

$$
h_{0}>0.5 \rho_{0} c_{0}^{2}(\sqrt{1+\alpha}-1),
$$

and equal or smaller otherwise. Therefore, the total damping coefficient standing by magnetoacoustic force of heating, may be smaller or larger than that in the absence of magnetic field. This depends on unperturbed magnetic pressure, $h_{0}$. The coefficient of diffusion in magnetic fluid is always larger. This suggests that the temperature gradients in the entropy mode decrease faster. The equation for the fourth mode follows from making use of $P_{4}$ :

$$
\begin{aligned}
& \frac{\partial \rho_{4}^{\prime}}{\partial t}=\gamma \rho_{0}\left(c_{m, 0}^{2}-c_{0}^{2}\right)\left(c_{m, 0}^{2} \delta_{1}+c_{0}^{2} \delta_{2}\right)\left(2 c _ { m , 0 } ^ { 6 } \left(\gamma c_{m, 0}^{2}\right.\right. \\
& \left.\left.-c_{0}^{2}(\gamma-1)\right)\right)^{-1}\left(\left(\frac{\partial v_{1}}{\partial x}\right)^{2}+v_{1} \frac{\partial^{2} v_{1}}{\partial x^{2}}\right) .
\end{aligned}
$$

The acoustic force of this mode equals zero in the absence of magnetic field, and its generation by the periodic or nearly periodic sound is ineffective. An impulse might weakly contribute in total density and total magnetic and acoustic pressures. Equation (23) is readily integrated over time for approximately progressive with the speed $c_{m, 0}$ magnetoacoustic perturbation with the result

$$
\begin{aligned}
\rho_{4}^{\prime} & =-\gamma \rho_{0}\left(c_{m, 0}^{2}-c_{0}^{2}\right)\left(c_{m, 0}^{2} \delta_{1}+c_{0}^{2} \delta_{2}\right)\left(2 c _ { m , 0 } ^ { 7 } \left(\gamma c_{m}^{2}\right.\right. \\
& \left.\left.-c_{0}^{2}(\gamma-1)\right)\right)^{-1} v_{1} \frac{\partial v_{1}}{\partial x} .
\end{aligned}
$$

Account of variance of shear viscosity with temperature does not affect the leading-order Eqs. (21), (23).

\section{Magnetoacoustic streaming in a two-dimensional flow}

We consider now velocity in the plane $(x, y)$, that is, $\boldsymbol{v}=\left(v_{x}(x, y, t), v_{y}(x, y, t), 0\right)$ perpendicular to magnetic field $\boldsymbol{H}=\left(0,0, H_{z}(x, y, t)\right)$. The effects relating to variability of thermal conductivity and shear viscosity are insignificant as for description of the vortex flow caused by sound in the leading order; they will be treated as constants in this section. The equations describing the magnetogasdynamic flow in two dimensions, take the leading-order form

$$
\begin{aligned}
& \frac{\partial \rho^{\prime}}{\partial t}+\rho_{0} \frac{\partial v_{x}}{\partial x}+\rho_{0} \frac{\partial v_{y}}{\partial y}=-\rho^{\prime} \frac{\partial v_{x}}{\partial x}-v_{x} \frac{\partial \rho^{\prime}}{\partial x}-\rho^{\prime} \frac{\partial v_{y}}{\partial y} \\
& -v_{y} \frac{\partial \rho^{\prime}}{\partial y} \\
& \frac{\partial v_{x}}{\partial t}-\frac{\delta_{1}}{4} \frac{\partial^{2} v_{x}}{\partial x^{2}}-\frac{\delta_{1}}{4} \frac{\partial^{2} v_{y}}{\partial x \partial y}-\frac{3 \delta_{1}}{4} \Delta v_{x} \\
& +\frac{1}{\rho_{0}} \frac{\partial\left(p^{\prime}+h^{\prime}\right)}{\partial x}=-(\boldsymbol{v} \cdot \nabla) v_{x}+\frac{\rho^{\prime}}{\rho_{0}^{2}} \frac{\partial\left(p^{\prime}+h^{\prime}\right)}{\partial x} \\
& -\frac{\delta_{1}}{4} \frac{\rho^{\prime}}{\rho_{0}} \frac{\partial}{\partial x} \nabla \cdot \boldsymbol{v}-\frac{3 \delta_{1}}{4} \frac{\rho^{\prime}}{\rho_{0}} \Delta v_{x}, \\
& \frac{\partial v_{y}}{\partial t}-\frac{\delta_{1}}{4} \frac{\partial^{2} v_{y}}{\partial y^{2}}-\frac{\delta_{1}}{4} \frac{\partial^{2} v_{x}}{\partial x \partial y}-\frac{3 \delta_{1}}{4} \Delta v_{y} \\
& +\frac{1}{\rho_{0}} \frac{\partial\left(p^{\prime}+h^{\prime}\right)}{\partial y}=-(\boldsymbol{v} \cdot \nabla) v_{y}+\frac{\rho^{\prime}}{\rho_{0}^{2}} \frac{\partial\left(p^{\prime}+h^{\prime}\right)}{\partial y} \\
& -\frac{\delta_{1}}{4} \frac{\rho^{\prime}}{\rho_{0}} \frac{\partial}{\partial y} \boldsymbol{\nabla} \cdot \boldsymbol{v}-\frac{3 \delta_{1}}{4} \frac{\rho^{\prime}}{\rho_{0}} \Delta v_{y},
\end{aligned}
$$




$$
\begin{aligned}
& \frac{\partial p^{\prime}}{\partial t}-\frac{\gamma \delta_{2}}{\gamma-1} \Delta p^{\prime}+\frac{\delta_{2} c_{0}^{2}}{\gamma-1} \Delta \rho^{\prime}+c_{0}^{2} \rho_{0} \nabla \cdot \boldsymbol{v}=-\boldsymbol{v} \cdot \nabla p^{\prime} \\
& -\gamma p^{\prime} \boldsymbol{\nabla} \cdot \boldsymbol{v}+\frac{3 \delta_{1}}{8}\left(\frac{\partial v_{i}}{\partial x_{k}}+\frac{\partial v_{k}}{\partial x_{i}}-\frac{2}{3} \delta_{i k} \frac{\partial v_{l}}{\partial x_{l}}\right)^{2} \\
& \quad-\frac{\delta_{2}}{\rho_{0}(\gamma-1)} \Delta\left(\gamma p^{\prime} \rho^{\prime}-c_{0}^{2} \rho^{\prime 2}\right), \\
& \frac{\partial h^{\prime}}{\partial t}+2 h_{0} \boldsymbol{\nabla} \cdot \boldsymbol{v}=-\boldsymbol{v} \cdot \boldsymbol{\nabla} h^{\prime}-2 h^{\prime} \boldsymbol{\nabla} \cdot \boldsymbol{v},
\end{aligned}
$$

where $x_{1}, x_{2}, x_{3}$ denote correspondingly $x, y$ and $z$, $\Delta=\partial^{2} / \partial x^{2}+\partial^{2} / \partial y^{2}$, and $i, k, l$ are integer numbers varying from 1 till 3 . The most important case is a weakly diffracting magnetoacoustic beam which propagates, for definiteness, in the positive direction of axis $x$. A small parameter may be introduced which accounts for diffraction and measures ratio of the characteristic scales of perturbations in the longitudinal and transversal directions, $\beta=k_{y} / k_{x}$, so that

$$
\sqrt{k_{x}^{2}+k_{y}^{2}} \approx k_{x}\left(1+\frac{k_{y}^{2}}{2 k_{x}^{2}}\right)
$$

etc. The expansion of dynamic equations in series with respect to powers of small parameters is a common procedure in nonlinear acoustics, this is a way to derive many simplified relations and equations, among them, the celebrated Khokhlov-Zabolotskaya-Kuznetsov equation, which describes propagation of weakly diffracting sound beam in a Newtonian fluid [19]. Considering small attenuation and diffraction, one readily arrives to the leading-order dispersion relations in a magnetogasdynamic flow,

$$
\begin{aligned}
& \omega_{1,2}= \pm c_{m, 0} k_{x}\left(1+\frac{k_{y}^{2}}{2 k_{x}^{2}}\right)+\frac{\mathrm{i}}{2}\left(\delta_{1}+\frac{c_{0}^{2}}{c_{m, 0}^{2}} \delta_{2}\right) k_{x}^{2} \\
& \omega_{3}=\mathrm{i}\left(\frac{\delta_{2}}{\gamma-1}+\frac{\delta_{2}\left(c_{m, 0}^{2}-c_{0}^{2}\right)}{c_{m, 0}^{2}}\right) k_{x}^{2}, \quad \omega_{4}=0 \\
& \omega_{5}=\mathrm{i} \frac{3}{4} \delta_{1} \Delta .
\end{aligned}
$$

The new root which appears in two-dimensional flow reflects existence of the incompressible rotational flow of a gas with velocity whose divergence is zero, $\boldsymbol{\nabla} \cdot \boldsymbol{v}_{5}=0$. The solenoidal velocity may be decomposed from the total one by applying of the operator $P_{\text {vor }}$ at the vector of the overall velocity

$$
\begin{gathered}
P_{\mathrm{vor}} \boldsymbol{v}=\Delta^{-1}\left(\begin{array}{cc}
\frac{\partial^{2}}{\partial y^{2}} & -\frac{\partial^{2}}{\partial x \partial y} \\
-\frac{\partial^{2}}{\partial x \partial y} & \frac{\partial^{2}}{\partial x^{2}}
\end{array}\right) \\
\times\left(\begin{array}{l}
\sum_{i=1}^{5} v_{x, i} \\
\sum_{i=1}^{5} v_{y, i}
\end{array}\right)=\left(\begin{array}{c}
v_{x, 5} \\
v_{y, 5}
\end{array}\right) .
\end{gathered}
$$

Applying $P_{\text {vor }}$ at the momentum equation (Eqs. (26), (27)), results in the equation governing magnetoacoustic streaming

$$
\frac{\partial \boldsymbol{v}_{5}}{\partial t}-\frac{3 \delta_{1}}{4} \Delta \boldsymbol{v}_{5}=-\frac{1}{\rho_{0}} P_{\mathrm{vor}}\left(\rho_{\mathrm{a}}^{\prime} \frac{\partial \boldsymbol{v}_{\mathrm{a}}}{\partial t}\right)=
$$

$$
\frac{c_{m, 0}}{2 \rho_{0}^{2}}\left(\delta_{1}+\frac{\delta_{2} c_{0}^{2}}{c_{m, 0}^{2}}\right) P_{\text {vor }} \rho_{\mathrm{a}}^{\prime} \nabla \frac{\partial}{\partial x}\left(\rho_{2}^{\prime}-\rho_{1}^{\prime}\right) .
$$

$\rho_{\mathrm{a}}^{\prime}, \boldsymbol{v}_{\mathrm{a}}$ denote summary acoustic perturbation in density, $\rho_{\mathrm{a}}^{\prime}=\sum_{i=1}^{2} \rho_{i}^{\prime}, \boldsymbol{v}_{\mathrm{a}}=\sum_{i=1}^{2} \boldsymbol{v}_{i}$. Equation (32) coincides with the instantaneous equation which governs acoustic streaming in Newtonian flows in the absence of magnetic field [21, 31]. Its averaged leading-order form in the case of the periodic magnetoacoustic wave, may be expressed in terms of magnetoacoustic pressure in the right-hand side,

$$
\begin{aligned}
& \frac{\overline{\partial v_{x, 5}}}{\partial t}-\frac{3 \delta_{1}}{4} \overline{\Delta v_{x, 5}}=F_{m, s}= \\
& \frac{1}{\rho_{0}^{2} c_{0}^{4} c_{m, 0}}\left(\delta_{1}+\delta_{2} \frac{c_{0}^{2}}{c_{m, 0}^{2}}\right) \overline{\left(\frac{\partial p_{1}^{\prime}}{\partial t}\right)^{2}} .
\end{aligned}
$$

This equation has the well-known limit in the case of non-magnetized Newtonian fluid. The details of the evaluations in the case of a Newtonian fluid may be found in Refs. [21, 31]. The leading-order equation which describes the longitudinal component of the vortex velocity $v_{x, 5}$ in the case of periodic acoustic pressure, takes the form [19, 32, 33]:

$$
\overline{\frac{\partial v_{x, 5}}{\partial t}}-\frac{3 \delta_{1}}{4} \overline{\Delta v_{x, 5}}=F_{N, s}=\frac{\delta_{1}+\delta_{2}}{\rho_{0}^{2} c_{0}^{5}} \overline{\left(\frac{\partial p_{1}^{\prime}}{\partial t}\right)^{2}} .
$$

The conclusion is that whereas the acoustic Newtonian force of streaming is determined by the summary attenuation due to irreversible mechanical and thermal losses, $\delta_{1}+\delta_{2}$, the acoustic force of magnetoacoustic streaming is always smaller than that in a Newtonian flow, $F_{m, s}<F_{N, s}$. Hence, the velocity of streaming is smaller as compared with a non-magnetized Newtonian fluid at equal Mach numbers.

\section{Concluding remarks}

This study brings out some features of the excitation of the non-wave modes by sound in the magnetogasdynamic flow. Weakly nonlinear governing equations of the nonwave modes are derived which take into account acoustic sources. They are valid for periodic and aperiodic sound. Equations (20), (21), (32), (33) are the main results of the study. They describe dynamics of magnetoacoustic heating and streaming independently of the spectrum of magnetoacoustic wave. The magnetoacoustic forces of heating and streaming, and hence, variations of the background temperature and mean streams depend on magnetic strength. These slow variations may be measured remotely, and the magnetic pressure in a plasma may be established analytically.

Magnetoacoustic heating in the case of periodic sound is determined by Eq. (21). Both Eqs. (21), (22) are inhomogeneous diffusity equations. Comparing the righthand sides of both equations, one may conclude that the acoustic force of magnetoacoustic heating $F_{m, h}$ may be smaller or larger than the Newtonian one $F_{N, h}$ in dependence on unperturbed magnetic pressure $h_{0}$. The magnetoacoustic force is not proportional to the 
summary attenuation, in contrast to $F_{N, h}$. The parameter of diffusity is larger in Eq. (21). In the multidimensional flow, nonlinear loss in acoustic momentum causes streaming. Equations (32), (33) govern twodimensional quasi-planar magnetoacoustic streaming and Newtonian streaming, respectively. The magnetoacoustic force of streaming is always smaller than the Newtonian one, $F_{m, s}<F_{N, s}$. In the right-hand sides of Eqs. (21), (22), partial derivative with respect to time may be used in the leading order; the acoustic forces may be also expressed in terms of partial derivative of acoustic pressure, recalling that

$$
\overline{\left(\frac{\partial v_{1}}{\partial x}\right)^{2}} \approx c_{m, 0}^{-2} \overline{\left(\frac{\partial v_{1}}{\partial t}\right)^{2}} \approx c_{m, 0}^{-4} \rho_{0}^{-2} \overline{\left(\frac{\partial p_{1}^{\prime}}{\partial t}\right)^{2}} .
$$

The equalities are approximate with regard to nonlinearity and attenuation. Magnetoacoustic forces of heating and streaming include only quadratic nonlinear terms, and they are proportional to the first powers of damping coefficients. The excitation of the Alfvén mode by sound is described by Eq. (23). It is insignificant. This concerns periodic, nearly periodic magnetoacoustic wave, and impulses.

There are not restrictions concerning strength of the magnetic field in this study. Equations (20), (32) are not averaged over the sound period, and they make use of instantaneous magnetoacoustic source. Magnetoacoustic perturbations may be periodic or not. Usually, nonlinear interactions are resolved by representing of interacting modes as a sum of harmonics and solving of coupling equations for exactly satisfied resonance conditions with some desired accuracy. As a rule, three-wave resonant interactions are considered $[15,16]$. The author applies the method of projecting of initial system of conservation equations into the system of coupling equations for interacting modes independently of their initial spectrum. The modified relations of perturbations in the progressive magnetosound wave are determined. They differ from that in the Riemann wave in the absence of magnetic field. These links describe the progressive wave more precisely and may be readily modified by involving terms of the higher order in series in powers of the Mach number. They may be useful in studies of nonlinear effects of a progressive magnetoacoustic wave and its nonlinear distortion in the course of propagation.

\section{References}

[1] N. Herlofson, Nature 165, 1020 (1950).

[2] C. Truesdell, Phys. Rev. 78, 823 (1950).

[3] N.S. Anderson, J. Acoust. Soc. Am. 25, 529 (1953).

[4] L.P. Singh, D.B.Singh, S. Ram, Applied Mathematics, 2, 653 (2011).

[5] V.D. Sharma, L.P. Singh, S. Ram, Phys. Fluids 30 , 1572 (1987).
[6] L.P. Singh, R. Singh, S.D. Ram, Astrophys. Space Sci. 342, 371 (2012).

[7] N. Geffen, Phys. Fluids 6, 566 (1963).

[8] E.A. Ponomarev, Sov. Astron. 5, 673 (1961).

[9] R.K. Anand, H.C. Yadav, Theor. Comp. Fluid Dyn. 28, 369 (2014).

[10] S.N. Ojha, A. Singh, Astrophys. Space Sci. 179, 45 (1991).

[11] V.D. Sharma, J. Sharma, Radhe Shyam, Acta Astronautica.8(8), 815 (1981).

[12] R.Z. Sagdeev, A.A. Galeev, Nonlinear Plasma Theory. Frontiers in Physics., Benjamin, New York 1969.

[13] V.I. Petviashvili, O.A. Pokhotelov, Solitary Waves in Plasmas and in the Atmosphere, Gordon and Breach, Berlin 1992.

[14] P.K. Shukla, L. Stenflo, in: Nonlinear MHD Waves and Turbulence, Lecture Notes in Solar Phys., Vol. 536, Eds. T. Passot, P.-L. Sulem, Springer, Berlin 1999.

[15] G. Brodin, L. Stenflo, P.K. Shukla, Solar Physics. 236(2), 285 (2006).

[16] D.I. Zavershinsky, N.E. Molevich, Techn. Phys. Lett. 40, 701 (2014).

[17] I. Ballai, J. Phys. Conf. Series 44, 20 (2006).

[18] S. Krishna Prasad, D. Banerjee, T. Van Doorsselaere, Astrophys. J. 789, 118 (2014).

[19] O.V. Rudenko, S.I. Soluyan, Theoretical Foundations of Nonlinear Acoustics, Plenum, New York 1977.

[20] M. Hamilton, D. Blackstock, Nonlinear Acoustics, Academic Press, New York 1998.

[21] A. Perelomova, Phys. Lett. A 357, 42 (2006).

[22] A. Perelomova, Acta Acustica United with Acustica 89, 754 (2003).

[23] A. Perelomova, Acta Phys. Pol. A 123, 681 (2013).

[24] V. P. Korobeinikov, Problems in the Theory Point Explosion in Gases, American Mathematical Society, Providence 1976.

[25] E.M. Lifshitz, L.P. Pitaevskii, A.M. Kosevich, Physical Kinetics, Vol. 10, Course of Theoretical Physics, Butterworth-Heinemann, Oxford 1981.

[26] A.C. Fabian, C.S. Reynolds, G.B. Taylor, R.J.H. Dunn, Monthly Notices R. Astron. Soc. 363, 891 (2005).

[27] L. Spitzer, Physics of Fully Ionized Gases, Wiley, New York 1962

[28] R. Chin, E. Verwichte, G. Rowlands, V.M. Nakariakov, Phys. Plasmas 17, 107 (2010).

[29] A. Perelomova, Ultrasonics 43, 95 (2004).

[30] S. Makarov, M. Ochmann, Acta Acustica United with Acustica 82, 579 (1996).

[31] A. Perelomova, P. Wojda, Acta Acustica United with Acustica 96, 807 (2010).

[32] V.E. Gusev, O.V. Rudenko, Sov. Phys. Acoust. 25, 493 (1979).

[33] V.E. Gusev, O.V. Rudenko, Sov. Phys. Acoust. 27, 481 (1980). 